\title{
SINGULAR SOLUTIONS AND ILL-POSEDNESS FOR THE EVOLUTION OF VORTEX SHEETS*
}

\author{
RUSSEL E. CAFLISCH $\dagger$ AND OSCAR F. ORELLANA $\ddagger$
}

\begin{abstract}
The evolution of a planar vortex sheet is described by the Birkhoff-Rott equation. Duchon and Robert [C.R. Acad. Sci. Paris, 302 (1986), pp. 183-186], [Comm. Partial Differential Equations, 13 (1988), pp. 1265-1295] have constructed exact solutions of this equation that are analytic for all $t<0$ but have a possible singularity in the curvature of the sheet at $t=0$. This shows that smooth initial data for a vortex sheet can lead to singularity formation at a finite time, in agreement with the results of numerical computation [J. Fluid Mech., 167 (1986), pp. 65-93], [J. Fluid Mech., 114 (1982), pp. 283-298] and of asymptotic expansion [Proc. Roy. Soc. London Ser. A, 365 (1979), pp. 105-119], [Theoretical and Applied Mechanics, in Proc. XVI Internat. Congr. Theoret. Appl. Mech., F. I. Niordson and N. Olhoff, eds., North-Holland, Amsterdam, 1984, pp. 629-633]. We present an independent construction of these solutions and use these results to infer that the vortex sheet problem is ill-posed in Sobolev class $H_{n}$ with $n>3 / 2$. Earlier results show well-posedness in an analytic function class [Comm. Pure Appl. Math., 39 (1986), pp. 807-838], [Comm. Math. Phys., 80 (1981), pp. 485-516]. Our method is to construct an explicit singular function that is a solution of the linearized equation, with a correction term added on to make the sum an exact solution of the nonlinear equation. The correction term is analyzed using the Cauchy-Kowalewski method.
\end{abstract}

Key words. vortex sheets, vorticity, Kelvin-Helmholtz instability, fluid dynamics, Birkhoff-Rott equation, singularities, ill-posedness, instability, Euler equations

AMS(MOS) classifications. 76C05, 96E30, 35L67

1. Introduction. A planar vortex sheet is a curve in a two-dimensional, inviscid, incompressible flow along which the fluid velocity is discontinuous. Vortex sheets, and the Kelvin-Helmholtz instability that they undergo, play an important role in many flows, such as mixing layers, two-fluid interfaces and flow past airfoils. Asymptotic analysis by Moore [11], [12] and numerical computation by Krasny [8] and Meiron, Baker, and Orszag [10] have shown that a vortex sheet may develop a singularity, i.e., infinite curvature at a point, in a finite time. The appearance of this singularity is important because it is immediately followed by rollup of the sheet [9]. A more mathematical reason for interest in such singularity formation is that it may serve as a simple analogue of singularity formation for the three-dimensional Euler equations.

Duchon and Robert $[6,16]$ perform a general construction of vortex sheet solutions that are analytic for all $t>0$, by choosing initial data to lie on the stable manifold for the Birkhoff-Rott equation ((1.1) below). The initial data can have singularities in the $(1+\nu)$ th derivative for any $\nu>0$ (for a precise statement see [6], [16]; fractional derivatives can be understood in the Hölder sense as in (1.6)). Our aim is to present an independent construction of these singular solutions and to discuss their significance. We also obtain slightly more precise pointwise information on the singularity, by using a pointwise norm rather than the Fourier norm in [6], [16].

The singular solutions found here or in [6], [16] can be used to construct exact solutions for vortex sheets that develop singularities at finite time starting from smooth

* Received by the editors August 17, 1987; accepted for publication (in revised form) June 3, 1988.

† Courant Institute of Mathematical Sciences, New York University, New York, New York 10012. The research of this author was supported in part by Air Force Office of Scientific Research grant AFOSR 85-0017 and University Research Initiative grant AFOSR 86-0352 and by the Alfred P. Sloan Foundation.

$\ddagger$ Universidad Técnica Federico Santa María, Valparaiso, Chile. The research of this author was supported in part by Fondo Nacional de Desarrollo Científico y Tecnológico (FONDECYT) under grant 235 and Universidad Técnica Federico Santa María, Valparaiso, Chile. 
initial data, as described in the concluding $\S 7$. This shows that for the two-dimensional Euler equations, singular initial data (a vortex sheet) can become more singular in finite time. Furthermore, the existence of such singular solutions shows the vortex sheet problem to be ill posed in Sobolev space $H^{n}$ for $n>3 / 2$. A somewhat different proof of ill-posedness for vortex sheets was given by Ebin [15].

The vortex sheet is parametrized by a real variable $\gamma$ defined so that it is Lagrangian (the value of $\gamma$ is constant on a given fluid particle) and so that the density of circulation with respect to the $\gamma$ variable on the sheet is 1 . The position of the vortex sheet is defined by a complex variable $z(\gamma, t)$ that satisfies the Birkhoff-Rott equation [1]:

$$
\frac{\partial}{\partial t} \bar{z}(\gamma, t)=\frac{1}{2 \pi i} P V \int_{-\infty}^{\infty} \frac{d \gamma^{\prime}}{z(\gamma, t)-z\left(\gamma^{\prime}, t\right)},
$$

in which the integral is a Cauchy principal value. An arbitrary constant in the definition of the Cauchy integral is irrelevant since the right-hand side involves a difference. Moreover, we will assume for simplicity that $z$ is odd in $\gamma$, i.e.,

$$
z(-\gamma, t)=-z(\gamma, t)
$$

so that $z(0, t)=0$. The function $z=\gamma$ is a steady solution of (1.1) and corresponds to a flat vortex sheet with a uniform density of circulation.

Linearization of (1.1) about the steady solution $z=\gamma$ yields the following equation for $z=\gamma+s$ :

$$
\partial_{t} \bar{s}(\gamma, t)=\frac{1}{2} H\left[s_{\gamma}\right]=\frac{1}{2}\left(s_{+\gamma}-s_{-\gamma}\right)
$$

in which $H$ is the Hilbert transform defined by $H[s]=s_{+}-s_{-}, s_{+}$is the upper analytic part of $s$, i.e., the part with positive Fourier wavenumbers, and $s_{-}$is the lower analytic part. The linearized equation (1.3) is unstable with modes $e^{i k \gamma \pm k t / 2}$. In this paper we are investigating the nonlinear behavior of this Kelvin-Helmholtz instability. Since the linearized modes have arbitrarily large temporal growth rates, we find that singularities may develop in finite time for the solutions of the nonlinear equation.

An explicit example of our singular solutions is

$$
\begin{gathered}
z(\gamma, t)=\gamma+s_{0}+r, \\
s_{0}(\gamma, t)=\varepsilon(1-i)\left\{\left(1-e^{-t / 2-i \gamma}\right)^{1+\nu}-\left(1-e^{-t / 2+i \gamma}\right)^{1+\nu}\right\}
\end{gathered}
$$

in which $\varepsilon$ is small. The dominant term $s_{0}$ is an exact solution of the linearized equation (1.3) and the correction term $r$ is negligible relative to $s_{0}$ as explained in Theorem 1. Since $s_{0} \simeq c \gamma^{1+\nu}$ for $t=0$ and $\gamma \simeq 0$, then $z_{\gamma \gamma} \simeq s_{0 \gamma \gamma} \simeq c \gamma^{\nu-1}$ (with a different constant c). Therefore the vortex sheet has an infinite curvature at $\gamma=0, t=0$ for $0<\nu<1$. For the approximate singular solution $z_{0}=\gamma+s_{0}$, with $s_{0}$ given by (1.5), the vortex strength $\left|z_{0 \gamma}\right|^{-1}(t=0)$ is plotted in Fig. 1. The cusp at $\gamma=0$ is due to the singularity. An explanation of the terms in (1.5) is as follows. The $e^{ \pm i \gamma}$ makes $s_{0}$ periodic and odd. The exponent $-t$ gives a solution that decays in time. Such a decaying solution requires the factors $(1-i)$ and $\frac{1}{2}$.

To define a more general class of functions $s_{0}$, let $\varepsilon$ be a small real number and let $1>\nu>\alpha>0$. For $s$ analytic in $|\operatorname{Im} \gamma|<\rho$ define the Hölder norm

$$
|s|_{\rho}=\sup _{|\operatorname{Im} \gamma|<\rho}|s(\gamma)|+\sup _{|\operatorname{Im} \gamma|,\left|\operatorname{Im} \gamma^{\prime}\right|<\rho} \frac{\left|s(\gamma)-s\left(\gamma^{\prime}\right)\right|}{\left|\gamma-\gamma^{\prime}\right|^{\alpha}} .
$$




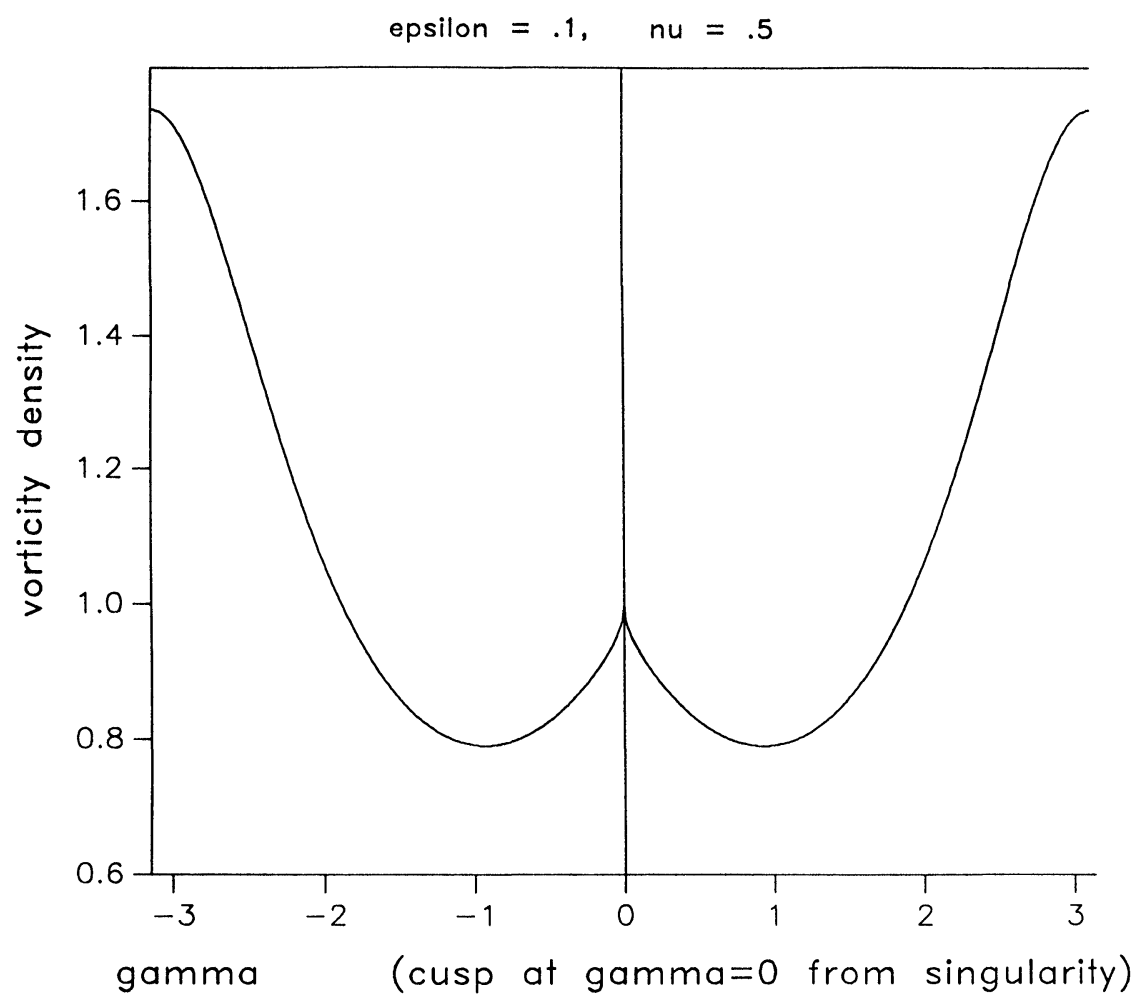

FIG. 1. Approximate vorticity density $\sigma=\left|z_{0 \gamma}\right|^{-1}=\left|1+s_{0 \gamma}\right|^{-1}$ with $s_{0}$ defined by (1.5), $\varepsilon=0.1, \nu=0.5$. The cusp at $\gamma=0$ corresponds to the singularity.

We require $s_{0}$ to satisfy the following:

(i) $s_{0}$ solves the linearized equation (1.3);

(ii) $s_{0}$ is analytic in the time-dependent strip $|\operatorname{Im} \gamma|<|t| / 2$ for $t>0$;

(iii) $s_{0}$ is small and decays to zero as $t \rightarrow \infty$. At $t=0 s_{0}$ has (at most) a singularity in its $(1+\nu)$ th derivative, i.e.,

$$
\begin{aligned}
& \left|s_{0}\right|_{\rho}+\left|s_{0 \gamma}\right|_{\rho}<c \varepsilon e^{-(|t| / 2-\rho)} \\
& \left|s_{0 \gamma \gamma}\right|_{\rho}<c \varepsilon\left(1+(|t|-2 \rho)^{\nu-\alpha-1}\right) e^{-(|t| / 2-\rho)}
\end{aligned}
$$

for $t>0$.

Note that the function $s_{0}$ in (1.5) satisfies (i)-(iii). Our main result is the following existence theorem.

THEOREM 1. Let $\varepsilon$ be a sufficiently small real number and let $1>\nu>\alpha>0$. Let $s_{0}$ satisfy (i)-(iii) above; i.e., $s_{0}$ is an analytic solution of the linearized equation (1.3) that decays to zero at $t=\infty$ and has a mild singularity at $t=0$. Then there is a function $r(\gamma, t)$ such that

$$
z(\gamma, t)=\gamma+s_{0}+r
$$

is an analytic solution of the Birkhoff-Rott equation (1.1) for $t>0$ and $\kappa|\operatorname{Im} \gamma|<t$ in which $\kappa>2$ and $\kappa \rightarrow 2$ as $\varepsilon \rightarrow 0$. Moreover, $r$ can be chosen so that the decaying mode $r_{+}+i \bar{r}_{-}=0$ at $t=0$ and that

$$
\begin{aligned}
& |r|_{0}+\left|r_{\gamma}\right|_{0}<c \varepsilon^{2} \exp (-|t| / 2), \\
& \left|r_{\gamma \gamma}\right|_{0}<c \varepsilon^{2}\left(1+|t|^{\nu-\alpha-1}\right) \exp (-|t| / 2)
\end{aligned}
$$

in which $c$ is some constant that is independent of $\varepsilon$ and depends smoothly on $\alpha^{-1}$ and $(\nu-\alpha)^{-1}$ (i.e., $c$ may be infinite at $\alpha=0$ or $\alpha=\nu$ ). 
Since $r_{+}+i \bar{r}_{-}=0$ at $t=0$, in some sense half of the initial data of $z$ is given by $\gamma+s_{0}$. The norm in (1.10), (1.11) is that of (1.6) with $\rho=0$. These estimates show that $r$ is as smooth as $s_{0}$, but much smaller. Stronger estimates for $r$ on the strip $\kappa|\operatorname{Im} \gamma|<t$ are given at the end of $\S 6$.

There are three main ideas in this construction: The first is to extend the BirkhoffRott equation to complex $\gamma$. Linearization (or equivalently the form of the KelvinHelmholtz instability) shows the Birkhoff-Rott equation to be approximately hyperbolic in the imaginary $\gamma$ direction, so that singularities will move in that direction. This was first pointed out by Moore [11]. Of course, only real values of $\gamma$ are physically meaningful; i.e., singularities are physically observable only when they lie on the real $\gamma$ line.

The second idea is to put the singularity in the initial data. By proper choice of initial data, the singularities can be expected to travel approximately on the lines Im $\gamma= \pm t / 2$. Although our method cannot track these singularities exactly, we are able to show that the resulting solution is analytic in the wedge $|\operatorname{Im} \gamma|<\kappa t / 2, t>0$, as shown in Fig. 2. Note that for all $t>0$ this wedge contains the line $\operatorname{Im} \gamma=0$. Therefore the vortex sheet is analytic for all $t>0$.

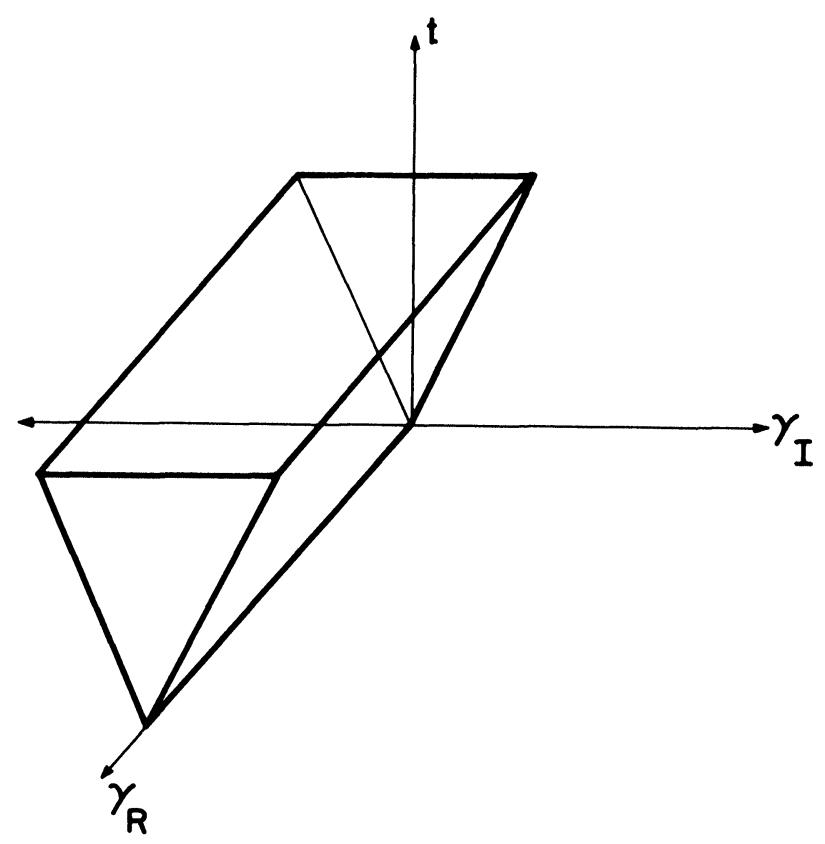

FIG. 2. Domain of analyticity (in $\gamma$ ) for $z(\gamma, t)$ (equivalently for $r(\gamma, t)$ ): $\{|\operatorname{Im} \gamma|<c t, t>0\}$.

The third idea is to construct the solution within the class of analytic functions. In this function class the Birkhoff-Rott equation has been shown to be well posed; while in almost any larger class it is expected to be ill posed. For the Sobolev spaces, ill-posedness is shown in $\S 7$. Use of analytic functions provides the stabilization necessary to construct exact solutions in the presence of physical instability. Aside from this practical justification, our belief that the imposition of analyticity is consistent with the zero viscosity limit justifies the use of analytic functions, at least for some flow regimes. 


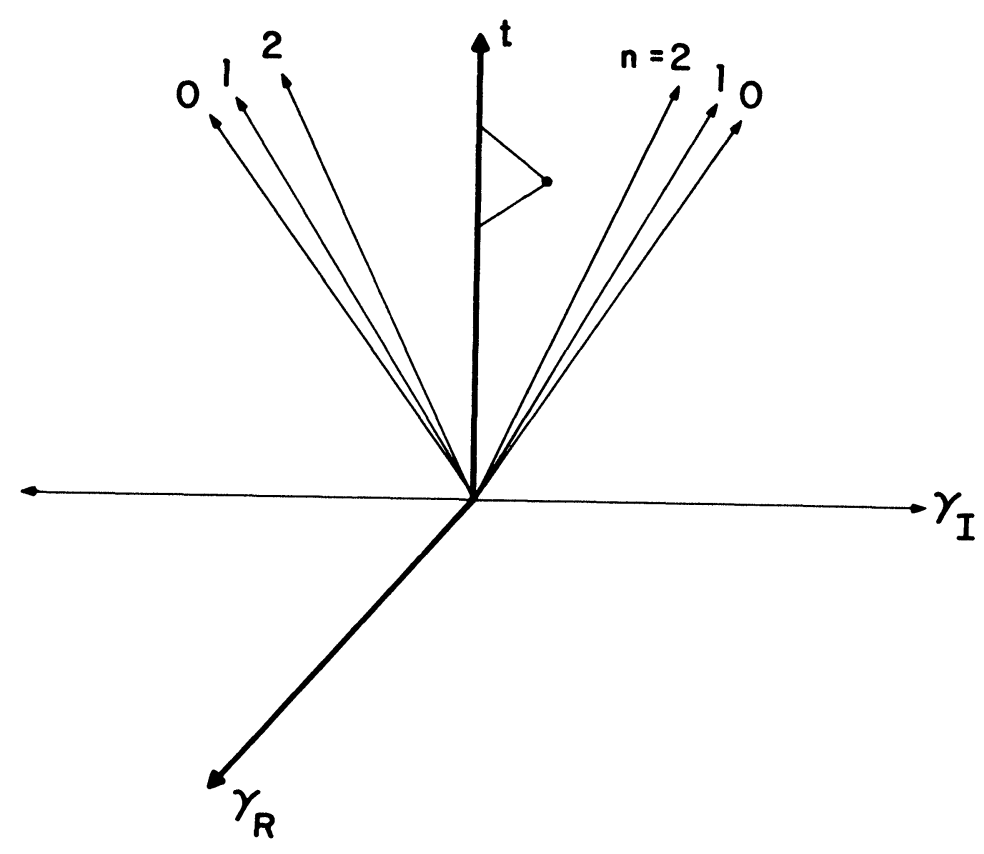

FIG. 3. Domain of analyticity for the iterates $r_{n}$ (or equivalently $p_{n}, q_{n}$ ). The width of the domain decreases as $n$ increases. The line segments emanating from the plane $\{\operatorname{Im} \gamma=0\}$ are the characteristics used to extend the iterates to complex $\gamma$, as in (2.12), (2.13), or (3.3).

The correction term $r$ of Theorem 1 is constructed using the Cauchy-Kowalewski method, somewhat modified from that in [13] involving an iteration. At each iteration in our construction, the approximate solution is analytic in a wedge $|\operatorname{Im} \gamma|<c t$, with an angle $c$ that is slightly reduced at each step, as indicated in Fig. 3. The final solution is valid in a limiting wedge, which is shown to be nonempty.

Other analytic results for vortex sheets include a proof of short-time existence by Sulem, Sulem, Bardos, and Frisch and a proof of existence almost up to the expected time of singularity formation by Caflisch and Orellana [2]. The singularities described in the present paper are not accompanied by concentrations of energy. Thus they are much weaker than those discussed by DiPerna and Majda [3]-[5], which possibly appear on a vortex sheet at a later time. On the other hand, there are no known examples for which such energy concentrations develop from less singular initial data.

The outline of this paper follows. Section 2 contains a reformulation of (1.1) and shows the sense in which the problem is hyperbolic. In particular, an equation for the error term $r$ is derived. In $\S 3$ we describe an iteration method for solving the equation for $r$. Each iteration involves solving an elliptic problem in $t$ and $\operatorname{Re} \gamma$, then extending the solution to complex $\gamma$ by solving a hyperbolic problem. The elliptic problem is solved using a Green's function; we solve the hyperbolic problem by integrating along characteristics. Estimates on the first iterate are obtained in $\S 4$, the induction method is described in $\S 5$ and estimates for the successive iterates are derived using a Cauchy-Kowalewski method in $\S 6$. At the end of that section, we summarize the proof of Theorem 1, and we derive two consequences of Theorem 1 in the concluding section.

2. Formulation. We write $z$ as a perturbation of the flat sheet by $z=\gamma+s$ and assume that $s$ is $2 \pi$-periodic, i.e.,

$$
s(\gamma+2 \pi, t)=s(\gamma, t) .
$$


The Birkhoff-Rott equation (1.1) is equivalent to the following equation for $s$, which is now written in a way that is analytic for complex $\gamma$,

$$
\frac{\partial}{\partial t} s^{*}(\gamma, t)=B[s] \equiv \frac{1}{2 \pi i} P V \int_{-\infty}^{\infty} \frac{d \zeta}{-\zeta+s(\gamma)-s(\gamma+\zeta)}
$$

in which $s^{*}$ is the analytic extension of $\bar{s}$, i.e., $s^{*}(\gamma)=\overline{s(\bar{\gamma})}$. The operator $B$ is expanded as $B[s]=B_{1}[s]+B_{r}[s]$ in which

$$
B_{1}[s](\gamma)=-\frac{1}{2 \pi i} P V \int_{-\infty}^{\infty} \frac{s(\gamma)-s(\gamma+\zeta)}{\zeta^{2}} d \zeta=\frac{1}{2} H\left[s_{\gamma}\right]
$$

is the linear part of $B$ and $B_{r}$ is the nonlinear remainder. The linearized equation (1.3) is analytically extended as $s_{t}^{*}=B_{1}[s]$.

Since $s_{0}$ is an exact solution of this linearized equation, the Birkhoff-Rott equation (1.1) for $z=\gamma+s_{0}+r$ can be rewritten as follows:

$$
r_{t}^{*}=B_{1}[r]+B_{r}\left[s_{0}+r\right] \text {. }
$$

Define the decaying component $p$ and growing component $q$ for $r$ as follows:

$$
p=r_{+}+i\left(r_{-}\right)^{*}, \quad q=r_{+}-i\left(r_{-}\right)^{*} .
$$

Then (2.4) can be rewritten as

$$
\begin{aligned}
& p_{t}=\frac{i}{2} p_{\gamma}+a, \\
& q_{t}=\frac{-i}{2} q_{\gamma}+b
\end{aligned}
$$

in which

$$
a=\left(B_{-}\right)^{*}+i B_{+}, \quad b=\left(B_{-}\right)^{*}-i B_{+}
$$

and $B_{+}$and $B_{-}$are the upper and lower analytic components of $B_{r}\left[s_{0}+r\right]$. Note that $a$ and $b$, and thus also $p$ and $q$, have only components with positive wave numbers.

The system (2.6), (2.7) is elliptic in $\gamma, t$ for real $\gamma$, but it is hyperbolic in $\gamma, t$ for imaginary $\gamma$ (or more precisely, in the imaginary $\gamma$ direction).

We solve (2.6), (2.7) in two parts. For $\gamma$ real, $t \geqq 0$, we solve the elliptic equation using a Green's function. For $\operatorname{Im} \gamma \neq 0, t \geqq 0$, we solve the hyperbolic equation (2.4) by integration along characteristics using the values of $r$ on $\operatorname{Im} \gamma=0$ as "initial values." These characteristics are indicated in Fig. 3.

We require $p$ and $q$ to be $2 \pi$-periodic, to vanish at $t=\infty$, and to have only components with positive wave numbers and $p=0$ at $t=0$; i.e.,

$$
\begin{gathered}
(p, q)(\gamma, t)=(p, q)(\gamma+2 \pi, t), \\
(p, q) \rightarrow 0 \text { as } t \rightarrow \infty, \quad p(t=0)=0, \\
(\hat{p}, \hat{q})(k, t)=\int_{0}^{2 \pi} \mathrm{e}^{-i k \gamma}(p, q)(\gamma, t) d \gamma=0 \quad \text { for } k \leqq 0 .
\end{gathered}
$$

Under these conditions and for $a$ and $b$ having only components with positive wave numbers, the solution of (2.6), (2.7) is uniquely given by

$$
\begin{aligned}
& p(\gamma, t)=\int_{0}^{t} \int_{0}^{2 \pi} g\left(\gamma^{\prime}, t-t^{\prime}\right) a\left(\gamma-\gamma^{\prime}, t^{\prime}\right) d \gamma^{\prime} d t^{\prime}, \\
& q(\gamma, t)=\int_{t}^{\infty} \int_{0}^{2 \pi} g\left(\gamma^{\prime}, t-t^{\prime}\right) b\left(\gamma-\gamma^{\prime}, t^{\prime}\right) d \gamma^{\prime} d t^{\prime}
\end{aligned}
$$


for $\gamma$ real and by

$$
\begin{aligned}
& p(\gamma+i \mu, t)=p(\gamma, t+2 \mu)-2 \int_{0}^{\mu} a\left(\gamma+i \mu^{\prime}, t+2\left(\mu-\mu^{\prime}\right)\right) d \mu^{\prime} \\
& q(\gamma+i \mu, t)=q(\gamma, t-2 \mu)+2 \int_{0}^{\mu} b\left(\gamma+i \mu^{\prime}, t-2\left(\mu-\mu^{\prime}\right)\right) d \mu^{\prime}
\end{aligned}
$$

for $\gamma+i \mu$ complex in which

$$
\begin{gathered}
g(\gamma, t)=\left\{\begin{aligned}
\xi(-t / 2+i \gamma), & t>0, \\
-\xi(-t / 2+i \gamma), & t<0,
\end{aligned}\right. \\
\xi(z)=\frac{1}{2 \pi} \frac{e^{z}}{1-e^{z}} .
\end{gathered}
$$

If $a$ and $b$ are analytic in $\gamma$, then $p$ and $q$ defined by (2.10)-(2.13) are also analytic. According to $(2.5), r=\frac{1}{2}(p+q)+i \frac{1}{2}\left(p^{*}-q^{*}\right)$. Let $A\left[s_{0}+r\right]$ denote the corresponding combination of the right-hand sides of (2.10)-(2.13). The Birkhoff-Rott equation (1.1) or (2.4) for $z=\gamma+s_{0}+r$ can be rewritten as follows:

$$
r=A\left[s_{0}+r\right] \text {. }
$$

Equations (2.10)-(2.13) for $p, q$ or the equivalent equation (2.16) for $r$ are the main results of this section.

3. Iteration method. In the previous section, the Birkhoff-Rott equation, for $z=$ $\gamma+s_{0}+r$ was reduced to (2.16) for $r$. We now solve this equation by iteration. Define $r_{0}=0$ and for $n \geqq 0$ let $r_{n+1}$ solve

$$
r_{n+1}(\gamma, t)=A\left[s_{0}+r_{n}\right]
$$

In terms of $p_{n}, q_{n}$, defined as in (2.5), equation (3.1) is written as in (2.10)-(2.13):

$$
\begin{gathered}
p_{n+1}(\gamma, t)=\int_{0}^{t} \int_{0}^{2 \pi} g\left(\gamma^{\prime}, t-t^{\prime}\right) a_{n}\left(\gamma-\gamma^{\prime}\right) d \gamma^{\prime} d t^{\prime}, \\
q_{n+1}(\gamma, t)=\int_{t}^{\infty} \int_{0}^{2 \pi} g\left(\gamma^{\prime}, t-t^{\prime}\right) b_{n}\left(\gamma-\gamma^{\prime}\right) d \gamma^{\prime} d t^{\prime}, \\
p_{n+1}(\gamma+i \mu, t)=p_{n+1}(\gamma, t+2 \mu)-2 \int_{0}^{\mu} a_{n}\left(\gamma+i \mu^{\prime}, t+2\left(\mu-\mu^{\prime}\right)\right) d \mu^{\prime}, \\
q_{n+1}(\gamma+i \mu, t)=q_{n+1}(\gamma, t-2 \mu)+2 \int_{0}^{\mu} b_{n}\left(\gamma+i \mu^{\prime}, t-2\left(\mu-\mu^{\prime}\right)\right) d \mu^{\prime}
\end{gathered}
$$

for $\gamma$ and $\mu$ real, in which $a_{n}, b_{n}$ are defined as in (2.8) with $r$ replaced by $r_{n}$. To show convergence of $r_{n}$ we obtain estimates on the difference

$$
R_{n}=r_{n}-r_{n-1} \text {. }
$$

Let $Q_{n}=q_{n}-q_{n-1}, P_{n}=p_{n}-p_{n-1}$. We use the following differentiated equations for $R_{n}$, or equivalently for $P_{n}, Q_{n}$ :

$$
\begin{aligned}
& \partial_{\gamma}^{k} P_{n+1}(\gamma, t)=\int_{0}^{t} \int_{0}^{2 \pi} g \partial_{\gamma}^{k}\left(a_{n}-a_{n-1}\right) d \gamma^{\prime} d t^{\prime}, \\
& \partial_{\gamma}^{k} Q_{n+1}(\gamma, t)=\int_{t}^{\infty} \int_{0}^{2 \pi} g \partial_{\gamma}^{k}\left(b_{n}-b_{n-1}\right) d \gamma^{\prime} d t^{\prime},
\end{aligned}
$$




$$
\begin{aligned}
& \partial_{\gamma}^{k} P_{n+1}(\gamma+i \mu, t)=\partial_{\gamma}^{k} P_{n+1}(\gamma, t+2 \mu)-2 \int_{0}^{\mu} \partial_{\gamma}^{k}\left(a_{n}-a_{n-1}\right) d \mu^{\prime} \\
& \partial_{\gamma}^{k} Q_{n+1}(\gamma+i \mu, t)=\partial_{\gamma}^{k} Q_{n+1}(\gamma, t-2 \mu)+2 \int_{0}^{\mu} \partial_{\gamma}^{k}\left(b_{n}-b_{n-1}\right) d \mu^{\prime}
\end{aligned}
$$

for $k=1,2$.

For the Hölder norm $|s|_{\rho}$ defined in (1.6), the Cauchy estimate for the derivative of an analytic function is

$$
\left|s_{\gamma}(\cdot, t)\right|_{\rho} \leqq\left(\rho^{\prime}-\rho\right)^{-1}|s(\cdot, t)|_{\rho^{\prime}} \quad \text { if } \rho^{\prime}>\rho .
$$

The nonlinear part $B_{r}$ of the Birkhoff-Rott integral operator is estimated as

$$
\begin{aligned}
& \left|B_{r}[s]\right|_{\rho} \leqq c_{0}\left|s_{\gamma}\right|_{\rho}^{2}, \\
& \left|B_{r}[s]_{\gamma}\right|_{\rho} \leqq c_{0}\left|s_{\gamma}\right|_{\rho}\left|s_{\gamma \gamma}\right|_{\rho}, \\
& \left|B_{r}[s]-B_{r}[\tilde{s}]\right|_{\rho} \leqq c_{1}\left|s_{\gamma}-\tilde{s}_{\gamma}\right|_{\rho}\left(\left|s_{\gamma}\right|_{\rho}+\left|\tilde{s}_{\gamma}\right|_{\rho}\right), \\
& \left|B_{r}[s]_{\gamma}-B_{r}[\tilde{s}]_{\gamma}\right|_{\rho} \leqq c_{1}\left|s_{\gamma}-\tilde{s}_{\gamma}\right|_{\rho}\left(\left|s_{\gamma \gamma}\right|_{\rho}+\left|\tilde{s}_{\gamma \gamma}\right|_{\rho}\right)+c_{1}\left|s_{\gamma \gamma}-\tilde{s}_{\gamma \gamma}\right|_{\rho}\left(\left|s_{\gamma}\right|_{\rho}+\left|\tilde{s}_{\gamma}\right|_{\rho}\right)
\end{aligned}
$$

for any $s, \tilde{s}$ in which $c_{0}=c\left(1-\left|s_{\gamma}\right|_{\rho}\right)^{-1}$ and $c_{1}=c\left(1-\left|s_{\gamma}\right|_{\rho}\right)^{-1}+c\left(1-\left|\tilde{s}_{\gamma}\right|_{\rho}\right)^{-1}$. For these estimates we assume that $\left|s_{\gamma}\right|_{\rho}<1,\left|\tilde{s}_{\gamma}\right|_{\rho}<1$.

From these general estimates it follows that $a_{n}, b_{n}$ satisfy

$$
\begin{gathered}
\left|a_{0}\right|_{\rho}+\left|b_{0}\right|_{\rho} \leqq c\left|s_{0 \gamma}\right|_{\rho}^{2}, \\
\left|a_{0 \gamma}\right|_{\rho}+\left|b_{0 \gamma}\right|_{\rho} \leqq c\left|s_{0 \gamma}\right|_{\rho}\left|s_{0 \gamma \gamma}\right|_{\rho}, \\
\left|a_{n}-a_{n-1}\right|_{\rho}+\left|b_{n}-b_{n-1}\right|_{\rho} \leqq c\left|R_{n \gamma}\right|_{\rho}\left(\left|s_{0 \gamma}\right|_{\rho}+\left|r_{n-1 \gamma}\right|_{\rho}+\left|r_{n \gamma}\right|_{\rho} \mid\right), \\
\left|\left(a_{n}-a_{n-1}\right)_{\gamma}\right|_{\rho}+\left|\left(b_{n}-b_{n-1}\right)_{\gamma}\right|_{\rho} \leqq c\left|R_{n \gamma}\right|_{\rho}\left(\left|s_{0 \gamma \gamma}\right|_{\rho}+\left|r_{n-1 \gamma \gamma}\right|_{\rho}+\left|r_{n \gamma \gamma}\right|_{\rho}\right) \\
+c\left|R_{n \gamma \gamma}\right|_{\rho}\left(\left|s_{0 \gamma}\right|_{\rho}+\left|r_{n-1 \gamma}\right|_{\rho}+\left|r_{n \gamma}\right|_{\rho}\right)
\end{gathered}
$$

if $\left|r_{m \gamma}\right|_{\rho}<\left|s_{0 \gamma}\right|_{\rho}<\frac{1}{2},\left|r_{m \gamma \gamma}\right|_{\rho}<\left|s_{0 \gamma \gamma}\right|_{\rho}<\frac{1}{2}$ for $m=n, n-1$.

For use in estimating the iterates $r_{n}$, we state a general lemma. Its proof is a straightforward extension of the proof of Hölder bounds on the Hilbert transform (Katznelson [7]).

LEMMA 3.1. Suppose that $\int_{0}^{2 \pi} a d \gamma=\int_{0}^{2 \pi} b d \gamma=0$ and let $p$ and $q$ satisfy

$$
\begin{aligned}
& p(\gamma, t)=\int_{0}^{t} \int_{0}^{2 \pi} g\left(\gamma^{\prime}, t-t^{\prime}\right) a\left(\gamma-\gamma^{\prime}, t^{\prime}\right) d \gamma^{\prime} d t^{\prime}, \\
& q(\gamma, t)=\int_{t}^{\infty} \int_{0}^{2 \pi} g\left(\gamma^{\prime}, t-t^{\prime}\right) b\left(\gamma-\gamma^{\prime}, t^{\prime}\right) d \gamma^{\prime} d t^{\prime}
\end{aligned}
$$

and suppose that

$$
\left|a_{\gamma}\right|_{0}+\left|b_{\gamma}\right|_{0} \leqq c \varepsilon^{2}\left(1+t^{\nu-\alpha-1}\right) e^{-t} .
$$

Let $r$ be related to $p$ and $q$ as in (2.5). Then $r$ satisfies

$$
\left|r_{\gamma}\right|_{0} \leqq c \varepsilon^{2} e^{-t / 2}, \quad\left|r_{\gamma \gamma}\right|_{0} \leqq c \varepsilon^{2}\left(1+t^{\nu-\alpha-1}\right) e^{-t / 2} .
$$

The same estimates are true for $p$ and $q$. 
4. First approximation. The first approximation $r_{1}=R_{1}$ satisfies $r_{1}=A\left[s_{0}\right]$, i.e.,

$$
\begin{gathered}
p_{1}(\gamma, t)=\int_{0}^{t} \int_{0}^{2 \pi} g a_{0} d \gamma^{\prime} d t^{\prime}, \quad q_{1}(\gamma, t)=\int_{t}^{\infty} \int_{0}^{2 \pi} g b_{0} d \gamma^{\prime} d t^{\prime}, \\
p_{1}(\gamma+i \mu, t)=p_{1}(\gamma, t+2 \mu)-2 \int_{0}^{\mu} a_{0} d \mu^{\prime}, \\
q_{1}(\gamma+i \mu, t)=q_{1}(\gamma, t-2 \mu)+2 \int_{0}^{\mu} b_{0} d \mu^{\prime}
\end{gathered}
$$

in which the arguments inside the integrals are as in (3.2)-(3.3). From (3.8), (1.7), and (1.8) it follows that $a_{0}, b_{0}$ satisfy

$$
\left|a_{0 \gamma}\right|_{\rho}+\left|b_{0 \gamma}\right|_{\rho} \leqq c \varepsilon^{2}\left(1+(t-2 \rho)^{\nu-\alpha-1}\right) e^{-(t-2 \rho)},
$$

for $t>2 \rho$. In particular,

$$
\left|a_{0 \gamma}\right|_{0}+\left|b_{0 \gamma}\right|_{0} \leqq c \varepsilon^{2}\left(1+t^{\nu-\alpha-1}\right) e^{-t} .
$$

Application of Lemma 3.1 implies

$$
\left|r_{1 \gamma}\right|_{0} \leqq c \varepsilon^{2} e^{-t / 2} \quad\left|r_{1 \gamma \gamma}\right|_{0} \leqq c \varepsilon^{2}\left(1+t^{\nu-\alpha-1}\right) e^{-t / 2} .
$$

Next estimate $r_{1 \gamma}$ for $\gamma+i \mu$ complex from (4.2) as

$$
\begin{aligned}
\left|r_{1 \gamma}(\cdot, t)\right|_{\rho} & \leqq 2 \sup _{|\mu| \leqq \rho}\left|r_{1 \gamma}(\cdot, t+2 \mu)\right|_{0}+2 \int_{0}^{\rho}\left|a_{0 \gamma}\left(t+2 \mu^{\prime}\right)\right|_{\rho-\mu^{\prime}}+\left|b_{0 \gamma}\left(t-2 \mu^{\prime}\right)\right|_{\rho-\mu^{\prime}} d \mu^{\prime} \\
& \leqq c \varepsilon^{2} e^{-(t-2 \rho) / 2}+c \varepsilon^{2} \rho\left(1+(t-2 \rho)^{\nu-\alpha-1}\right) e^{-(t-2 \rho)} \\
& \leqq c \delta^{\nu-\alpha-1} \varepsilon^{2} e^{-(t-2 \rho) / 2}
\end{aligned}
$$

for $\kappa_{1} \rho<t$ with $\kappa_{1}=2(1+\delta)$ with $\delta>0$. Similarly,

$$
\begin{aligned}
\left|r_{1 \gamma \gamma}(\cdot, t)\right|_{\rho} \leqq & 2 \sup _{|\mu| \leqq \rho}\left|r_{1 \gamma \gamma}(\cdot, t+2 \mu)\right|_{0}+2 \int_{0}^{\rho}\left|a_{0 \gamma \gamma}\left(t+2 \mu^{\prime}\right)\right|_{\rho-\mu^{\prime}}+\left|b_{0 \gamma \gamma}\left(t-2 \mu^{\prime}\right)\right|_{\rho-\mu^{\prime}} d \mu^{\prime} \\
\leqq & c \varepsilon^{2}\left(1+(t-2 \rho)^{\nu-\alpha-1}\right) e^{-(t-2 \rho) / 2} \\
& +(\delta \rho)^{-1} \int_{0}^{\rho}\left(\left|a_{0 \gamma}\right|_{(1+\delta) \rho-\mu^{\prime}}+\left|b_{0 \gamma}\right|_{(1+\delta) \rho-\mu^{\prime}}\right) d \mu^{\prime} \\
\leqq & c \varepsilon^{2}\left(1+(t-2 \rho)^{\nu-\alpha-1}\right) e^{-(t-2 \rho) / 2}+2 \varepsilon^{2} \delta^{-1}\left(1+\left(t-\kappa_{1} \rho\right)^{\nu-\alpha-1}\right) e^{-\left(t-\kappa_{1} \rho\right) / 2} \\
\leqq & c \delta^{-1} \varepsilon^{2}\left(1+\left(t-\kappa_{1} \rho\right)^{\nu-\alpha-1}\right) e^{-\left(t-\kappa_{1} \rho\right) / 2}
\end{aligned}
$$

for $\kappa_{1} \rho<t$.

5. Induction hypothesis. Successive approximations $r_{n}$ and their convergence are analyzed using an analogue of the Cauchy-Kowalewski method [13] for the integral equations (3.2), (3.3). In each iteration the wedge of existence $|\operatorname{Im} \gamma|<\kappa_{n}^{-1} t$ is slightly decreased, so that the general estimate (3.6) can be employed. The object of the modified Cauchy-Kowalewski method is to show that there is a limiting, nonempty wedge in which the solution $r$ is analytic. The key point of the method is overcoming the large factor $\left(\rho-\rho^{\prime}\right)^{-1}$ in the Cauchy estimate (3.6). As a result of this factor the singularity in the solution $r$ may be one order higher than the singularity in $s_{0}$ on the lines $|\operatorname{Im} \gamma|=\kappa_{n}^{-1} t$. However, the additional singularity has amplitude proportional to $t$, so that at $t=0, r$ is no more singular than $s_{0}$. 
The induction argument follows that of Nishida [13] with some change in notation and indexing. Define

$$
\kappa_{n+1}=\kappa_{n}\left(1-\varepsilon^{\mu}(n+2)^{-2}\right)^{-1}
$$

for $n \geqq 1$, in which $\mu>0$ is to be chosen later. Then

$$
\kappa_{n} \rightarrow \kappa=\kappa_{1} \prod_{1}^{\infty}\left(1-\varepsilon^{\mu}(m+2)^{-2}\right)^{-1},
$$

which is finite and note that $\kappa_{n+1}>\kappa_{n}>\cdots>\kappa_{1}>2$. Define the norm

$$
\begin{aligned}
A_{m}(R)= & \sup _{t>\kappa_{m} \rho} \varepsilon^{-2} e^{\left(t-\kappa_{m} \rho\right) / 2} t^{-1}\left(t-\kappa_{m} \rho\right) \\
& \times\left\{\left|R_{\gamma}(t)\right|_{\rho}+\left(1+\left(t-\kappa_{m} \rho\right)^{\nu-\alpha-1}\right)^{-1}\left|R_{\gamma \gamma}(t)\right|_{\rho}\right\}
\end{aligned}
$$

Since $\kappa_{m+1}>\kappa_{m}$, then $A_{m+1}(R) \leqq A_{m}(R)$ for any $R$. The size of the $m$ th correction $R_{m}=r_{m}-r_{m-1}$ is measured by

$$
\lambda_{m}=A_{m}\left(R_{m}\right) .
$$

As mentioned above, the factor $\left(t-\kappa_{m} \rho\right) / t$ indicates possible additional singularity on the line $|\operatorname{Im} \gamma|=\rho=\kappa_{m}^{-1} t$, but note that it does not affect the size of $R_{m}$ on the physical line $\operatorname{Im} \gamma=\rho=0$.

The induction hypothesis is

$$
\sum_{j=1}^{n} \lambda_{j}\left(1-\frac{\kappa_{j}}{\kappa_{j+1}}\right) \leqq d .
$$

The constant $d$ as well as $\kappa_{1}$ will be chosen later. In the previous section, we showed that $\lambda_{1} \leqq c\left(\kappa_{1}-2\right)^{-1}$ so that the hypothesis for $n=1$ is satisfied if $d \geqq c\left(\kappa_{1}-2\right)^{-1}$.

Now suppose that (5.5) is true for $n \geqq 1$. If $\kappa_{n+1} \rho<t$, then for $n \geqq j \geqq 1$,

$$
\begin{gathered}
\left|R_{j \gamma}(t)\right|_{\rho}<\lambda_{j}\left(1-\frac{\kappa_{j}}{\kappa_{n+1}}\right)^{-1} \varepsilon^{2} e^{-\left(t-\kappa_{j} \rho\right) / 2} \\
<\lambda_{j}\left(1-\frac{\kappa_{j}}{\kappa_{j+1}}\right)^{-1} \varepsilon^{2} e^{-\left(t-\kappa_{n} \rho\right) / 2} \\
\left|R_{j \gamma \gamma}(t)\right|_{\rho}<\lambda_{j}\left(1-\frac{\kappa_{j}}{\kappa_{n+1}}\right)^{-1} \varepsilon^{2}\left(1+\left(t-\kappa_{j} \rho\right)^{\nu-\alpha-1}\right) e^{-\left(t-\kappa_{j} \rho\right) / 2} \\
<\lambda_{j}\left(1-\frac{\kappa_{j}}{\kappa_{j+1}}\right)^{-1} \varepsilon^{2}\left(1+\left(t-\kappa_{n} \rho\right)^{\nu-\alpha-1}\right) e^{-\left(t-\kappa_{n} \rho\right) / 2} .
\end{gathered}
$$

Since $r_{0}=0, r_{n \gamma}=\sum_{j=1}^{n} R_{j \gamma}, r_{n \gamma \gamma}=\sum_{j=1}^{n} R_{j \gamma \gamma}$, and using the induction hypothesis at $n$, this yields

$$
\begin{aligned}
\left|r_{j \gamma}(t)\right|_{\rho} & \leqq \varepsilon^{2}\left(\sum_{j=1}^{n} \lambda_{j}\left(1-\kappa_{j} / \kappa_{j+1}\right)^{-1}\right) e^{-\left(t-\kappa_{n} \rho\right) / 2} \\
& \leqq d \varepsilon^{2} e^{-\left(t-\kappa_{n} \rho\right) / 2}, \\
\left|r_{j \gamma \gamma}(t)\right|_{\rho} & \leqq d \varepsilon^{2}\left(1+\left(t-\kappa_{n} \rho\right)^{\nu-\alpha-1}\right) e^{-\left(t-\kappa_{n} \rho\right) / 2},
\end{aligned}
$$

for any $j \leqq n$ and $\kappa_{n+1} \rho<t$. The bounds (5.8), (5.9) will be used in $\S 6$ for estimation of $R_{n+1}$. 
6. Successive approximations. To verify the induction hypothesis (5.5) for $n+1$, the correction terms $R_{n+1}$ that solve (3.4), (3.5) must be estimated. Using (1.7), (1.8) for $s_{0},(5.8)$, (5.9) for $r_{n}, r_{n-1}$, and (5.4) for $R_{n \gamma}, R_{n \gamma \gamma}$ in (3.10), the forcing term in (3.4), (3.5) is bounded for $\kappa_{n+1} \rho<t$ by

$$
\begin{aligned}
& \left|\left(a_{n}-a_{n-1}\right)_{\gamma}\right|_{\rho}+\left|\left(b_{n}-b_{n-1}\right)_{\gamma}\right|_{\rho} \\
& \quad \leqq c \varepsilon^{3}(1+\varepsilon d) \lambda_{n}\left(\frac{t}{t-\kappa_{n} \rho}\right)\left(1+\left(t-\kappa_{n} \rho\right)^{\nu-\alpha-1}\right) e^{-\left(t-\kappa_{n} \rho\right)} .
\end{aligned}
$$

In particular for $\rho=0$,

$$
\left|\left(a_{n}-a_{n-1}\right)_{\gamma}\right|_{0}+\left|\left(b_{n}-b_{n-1}\right)_{\gamma}\right|_{0} \leqq c \varepsilon^{3}(1+\varepsilon d) \lambda_{n}\left(1+t^{\nu-\alpha-1}\right) e^{-t} .
$$

First, estimate $R_{n+1}$ for $\gamma$ real, i.e., $\rho=0$. Application of Lemma 3.1 to (3.4) using (6.2) implies that

$$
\begin{aligned}
& \left|R_{n+1 \gamma}\right|_{0} \leqq c \varepsilon^{3}(1+\varepsilon d) \lambda_{n} e^{-t / 2}, \\
& \left|R_{n+1 \gamma \gamma}\right|_{0} \leqq c \varepsilon^{3}(1+\varepsilon d) \lambda_{n}\left(1+t^{\nu-\alpha-1}\right) e^{-t / 2}
\end{aligned}
$$

Second, estimate $R_{n+1 \gamma}$ for complex $\gamma+i \mu$, i.e., for $\rho \geqq 0$, solving (3.5). For $\kappa_{n+1} \rho<t$ bound

$$
\begin{aligned}
\left|R_{n+1 \gamma}(t)\right|_{\rho} \leqq 2 \sup _{|\mu|<\rho}\left|R_{n+1 \gamma}(t+2 \mu)\right|_{0} & +\int_{0}^{\rho}\left(\left|\left(a_{n}-a_{n-1}\right)_{\gamma}(t+2 \mu)\right|_{\rho-\mu}\right. \\
& \left.+\left|\left(b_{n}-b_{n-1}\right)_{\gamma}(t-2 \mu)\right|_{\rho-\mu}\right) d \mu \\
\leqq & 2 c \varepsilon^{3}(1+\varepsilon d) \lambda_{n} e^{-(t-\rho) / 2}+I
\end{aligned}
$$

in which $I$ denotes the integral. The inequalities $\kappa_{n+1}>\kappa_{n}>2$ and $\kappa_{n+1} \rho<t$ imply $\kappa_{n+1}(\rho-\mu)<(t-2 \mu)$. Then we may use (6.1) to bound $I$ by

$$
\begin{gathered}
I \leqq c \varepsilon^{3}(1+\varepsilon d) \lambda_{n} \int_{0}^{\rho}\left(\frac{t \pm 2 \mu}{(t \pm 2 \mu)-\kappa_{n}(\rho-\mu)}\right)\left(1+\left(t \pm 2 \mu-\kappa_{n}(\rho-\mu)\right)^{\nu-\alpha-1}\right) \\
\cdot e^{-\left(t \pm 2 \mu-\kappa_{n}(\rho-\mu)\right)} d \mu
\end{gathered}
$$

$$
\begin{aligned}
& \leqq c \varepsilon^{3}(1+\varepsilon d) \lambda_{n}(t+\rho) e^{-(3 / 4)\left(t-\kappa_{n} \rho\right)} \int_{0}^{\rho}\left(t-\kappa_{n} \rho+\left(\kappa_{n}-2\right) \mu\right)^{\nu-\alpha-2} d \mu \\
& \leqq c \varepsilon^{3}(1+\varepsilon d)\left(\kappa_{n}-2\right)^{-1} \lambda_{n}(t+\rho)\left(t-\kappa_{n} \rho\right)^{\nu-\alpha-1} e^{-(3 / 4)\left(t-\kappa_{n} \rho\right)} \\
& \leqq c \varepsilon^{3}(1+\varepsilon d)\left(\kappa_{n}-2\right)^{-1} \lambda_{n}\left(\frac{t}{t-\kappa_{n} \rho}\right) e^{-\left(t-\kappa_{n} \rho\right) / 2}
\end{aligned}
$$

Since $\kappa_{n+1}>\kappa_{n}$, this combines with (6.5) to show that

$$
\left|R_{n+1 \gamma}(t)\right|_{\rho} \leqq c \varepsilon^{3}(1+\varepsilon d)\left(\kappa_{n}-2\right)^{-1} \lambda_{n}\left(\frac{t}{t-\kappa_{n+1} \rho}\right) e^{-\left(t-\kappa_{n+1} \rho\right) / 2},
$$

for $\kappa_{n+1} \rho<t$.

Third, estimate $R_{n+1 \gamma \gamma}$ for complex $\gamma+i \mu$, i.e., for $\rho \geqq 0$, solving (3.5). This is the crucial estimate of the Cauchy-Kowalewski method. For $\kappa_{n+1} \rho<t$ estimate

$$
\begin{aligned}
\left|R_{n+1 \gamma \gamma}(t)\right|_{\rho} \leqq 2 \sup _{|\mu| \leqq \rho}\left|R_{n+1 \gamma \gamma}(t+2 \mu)\right|_{0} \\
\quad+\int_{0}^{\rho}\left\{\left|\left(a_{n}-a_{n-1}\right)_{\gamma \gamma}(t+2 \mu)\right|_{\rho-\mu}+\left|\left(b_{n}-b_{n-1}\right)_{\gamma \gamma}(t-2 \mu)\right|_{\rho-\mu}\right\} d \mu \\
\leqq c \varepsilon^{3}(1+\varepsilon d) \lambda_{n}\left(1+(t-2 \rho)^{\nu-\alpha-1}\right) e^{-(t-2 \rho) / 2}+I_{2},
\end{aligned}
$$


in which $I_{2}$ is the integral. Define $\rho_{1}$ by

$$
\rho_{1}=\frac{1}{2}\left(\kappa_{n+1}^{-1}(t-2 \mu)+(\rho-\mu)\right)
$$

which satisfies

$$
\begin{gathered}
\rho-\mu<\rho_{1}<\kappa_{n+1}^{-1}(t-2 \mu), \\
t-2 \mu-\kappa_{n+1} \rho_{1}=\kappa_{n+1}\left(\rho_{1}-(\rho-\mu)\right)=\left(t-\kappa_{n+1} \rho+\left(\kappa_{n+1}-2\right) \mu\right) / 2 \\
\geqq\left(t-\kappa_{n+1} \rho\right) / 2 .
\end{gathered}
$$

Estimate $I_{2}$ using the Cauchy estimate (3.6), the bound (6.1), and the relations (6.10), (6.11) to obtain

$$
\begin{aligned}
& I_{2} \leqq 2 \int_{0}^{\rho}\left(\rho_{1}-(\rho-\mu)\right)^{-1}\left|\left(a_{n}-a_{n-1}\right)_{\gamma}(t+2 \mu)\right|_{\rho_{1}}+\left|\left(b_{n}-b_{n-1}\right)_{\gamma}(t-2 \mu)\right|_{\rho_{1}} d \mu \\
& \leqq c \varepsilon^{3}(1+\varepsilon d) \lambda_{n} \int_{0}^{\rho}\left(\rho_{1}-(\rho-\mu)\right)^{-1} \frac{t+2 \mu}{\left(t-2 \mu-\kappa_{n} \rho_{1}\right)}\left(1+\left(t-2 \mu-\kappa_{n} \rho_{1}\right)^{\nu-\alpha-1}\right) \\
& \quad \cdot e^{-\left(t-2 \mu-\kappa_{n+1} \rho_{1}\right)} d \mu \\
& \leqq c \varepsilon^{3}(1+\varepsilon d) \lambda_{n}(t+\rho) e^{-\left(t-\kappa_{n+1} \rho\right) / 2} \int_{0}^{\rho}\left(t-\kappa_{n+1} \rho+\left(\kappa_{n+1}-2\right) \mu\right)^{-2} \\
& \quad+\left(t-\kappa_{n+1} \rho+\left(\kappa_{n+1}-2\right) \mu\right)^{\nu-\alpha-3} d \mu \\
& \leqq c \varepsilon^{3}(1+\varepsilon d) \lambda_{n}(t+\rho) e^{-\left(t-\kappa_{n+1} \rho\right) / 2}\left(\kappa_{n+1}-2\right)^{-1} \\
& \quad \cdot\left(t-\kappa_{n+1} \rho\right)^{-1}\left(1+\left(t-\kappa_{n+1} \rho\right)^{\nu-\alpha-1}\right) .
\end{aligned}
$$

Combine this with (6.8) to find

$$
\begin{array}{r}
\left|R_{n+1 \gamma \gamma}\right|_{\rho} \leqq c \varepsilon^{3}(1+d) \lambda_{n}\left(\kappa_{n+1}-2\right)^{-1}\left(\frac{t}{t-\kappa_{n+1} \rho}\right) \\
\cdot\left(1+\left(t-\kappa_{n+1} \rho\right)^{\nu-\alpha-1}\right) e^{-\left(t-\kappa_{n+1} \rho\right) / 2}
\end{array}
$$

for $\kappa_{n+1} \rho<t$.

Inequalities (6.7) for $R_{n+1 \gamma}$ and (6.13) for $R_{n+1 \gamma \gamma}$ and the definition (5.4) of $\lambda_{n+1}$ imply that

$$
\begin{aligned}
\lambda_{n+1} & \leqq c \varepsilon(1+\varepsilon d)\left(\kappa_{n+1}-2\right)^{-1} \lambda_{n} \\
& \leqq c \varepsilon(1+\varepsilon d)\left(\kappa_{1}-2\right)^{-1} \lambda_{n} .
\end{aligned}
$$

This inequality is also true for $\lambda_{j+1}$ in terms of $\lambda_{j}$ for any $j \leqq n$, so that

$$
\begin{aligned}
\lambda_{n+1} & \leqq\left\{c \varepsilon(1+\varepsilon d)\left(\kappa_{1}-2\right)^{-1}\right\}^{n} \lambda_{1} \\
& \leqq\left\{c \varepsilon(1+\varepsilon d)\left(\kappa_{1}-2\right)^{-1}\right\}^{n}\left(\kappa_{1}-2\right)^{-1} .
\end{aligned}
$$

With these estimates finished, we are ready to verify the induction hypothesis (5.5) for $n+1$ by choosing $d$ and $\kappa_{1}$. Let

$$
\kappa_{1}=2+a \varepsilon^{\mu}, \quad d=\varepsilon^{-2 \mu}
$$

in which $a$ and the parameters $\mu$ from (5.1) are still to be chosen. 


\section{Estimate}

$$
\begin{aligned}
\sum_{j=1}^{n+1} \lambda_{j}\left(1-\kappa_{j} / \kappa_{j+1}\right)^{-1} & \leqq\left(\kappa_{1}-2\right)^{-1} \sum_{j=1}^{\infty}\left(c \varepsilon(1+\varepsilon d)\left(\kappa_{1}-2\right)^{-1}\right)^{j-1}\left(1-\kappa_{j} / \kappa_{j+1}\right)^{-1} \\
& =a^{-1} \varepsilon^{-2 \mu} \sum_{j=1}^{\infty}\left(c a^{-1} \varepsilon^{1-\mu}\left(1+\varepsilon^{1-2 \mu}\right)\right)^{j-1}(j+2)^{2} \\
& <\varepsilon^{-2 \mu}
\end{aligned}
$$

for any $\mu$ with $0 \leqq \mu \leqq 2 / 3$ and a chosen (independently of $\mu$ ) to be sufficiently large. This verifies the induction hypothesis $(5.5)$ for $(n+1)$, for any $n$.

Completion of the induction proof shows that the inequalities (5.6), (5.7) on the $j$ th corrections $R_{j}$ are valid for all $j$ and that therefore the approximate solutions $r_{n}$ have a limit $r$ that solves (2.16). Finally the combination $z(\gamma, t)=\gamma+s_{0}(\gamma, t)+r(\gamma, t)$ solves the Birkhoff-Rott equation (1.1).

Moreover, the solution $r=\lim _{n \rightarrow \infty} r_{n}$ is analytic in $\kappa \rho<t$ with

$$
\begin{aligned}
\kappa & =\lim _{m \rightarrow \infty} \kappa_{m}=\kappa_{1} \Pi\left(1-\varepsilon^{\mu}(m+2)^{2}\right)^{-1} \\
& =2+\underline{O}\left(\varepsilon^{\mu}\right) .
\end{aligned}
$$

The bounds (5.8), (5.9) show that for $\kappa \rho<t, r$ satisfies

$$
\begin{aligned}
& |r(t)|_{\rho}+\left|r_{\gamma}(t)\right|_{\rho} \leqq c \varepsilon^{2-2 \mu} e^{-(t-\kappa \rho) / 2} \\
& \left|r_{\gamma \gamma}(t)\right|_{\rho} \leqq c \varepsilon^{2-2 \mu}\left(1+(t-\kappa \rho)^{\nu-\alpha-1}\right) e^{-(t-\kappa \rho) / 2} .
\end{aligned}
$$

By choosing $\mu=\frac{2}{3}$, its largest permissible size, we find that $r$ is analytic in the region $\left(2+Q\left(\varepsilon^{2 / 3}\right)\right) \rho<t$. By choosing $\mu=0$, we obtain the optimal bounds on the size of $r$, although on a restricted domain. In particular for $\rho=0$, i.e., on the physical line $\gamma$ real, the bounds are

$$
\begin{aligned}
& |r(t)|_{0}+\left|r_{\gamma}(t)\right|_{0} \leqq c \varepsilon^{2} e^{-t / 2} \\
& \left|r_{\gamma \gamma}(t)\right|_{0} \leqq c \varepsilon^{2}\left(1+t^{\nu-\alpha-1}\right) e^{-t / 2} .
\end{aligned}
$$

This completes the proof of Theorem 1 .

7. Conclusions. We will use Theorem 1 to derive solutions of the Birkhoff-Rott equation that develop singularities (i.e., infinite curvature) at finite time starting from analytic initial data. Then we show that the initial value problem for this equation is ill posed in Sobolev space $H^{n}\left(n>\frac{3}{2}\right)$, since the derivative of order $1+\nu$, for any $\nu>0$, can become infinite in an arbitrarily small time from arbitrarily small initial data (fractional derivatives are understood in the Hölder sense). On the other hand, the vortex sheet problem is known to be well posed in an analytic function setting for at least a short time [2], [14]. DiPerna and Majda [3]-[5] address the related question of finding an appropriate function space that is preserved by the Euler flow and by limits of Euler solutions, as well as by limits of regularized solutions (i.e., of the Navier-Stokes equations or the numerical vortex method).

The Birkhoff-Rott equation has the following three symmetry properties: If $z(\gamma, t)$ is a solution of $(1.1)$ then so are $z_{b}(\gamma, t)=z^{*}(\gamma,-t), z_{s}(\gamma, t)=z\left(\gamma, t-t_{0}\right)$, and $z_{n}(\gamma, t)=$ $n^{-1} z(n \gamma, n t)$.

When we use $z_{b}$, Corollary 1 follows from Theorem 1.

Corollary 1. Let $\varepsilon, \nu, \alpha$ be as in Theorem 1 and let $s_{0}$ satisfy properties (i)-(iii) except for $t<0$; i.e., $s_{0}$ is an analytic solution of the linearized equation (1.3) which decays to zero at $t=-\infty$ (decaying backwards in time) and has a mild singularity at $t=0$. Then 
there is a function $r(\gamma, t)$ such that $z(\gamma, t)=\gamma+s_{0}+r$ is an analytic solution of the Birkhoff-Rott equation (1.1) for $t<0$ and $\kappa|\operatorname{Im} \gamma|<|t|$ in which $\kappa>2$ and $\kappa \rightarrow 2$ as $\varepsilon \rightarrow 0$. Moreover, $r$ can be chosen so that the backward decaying mode $r_{+}-i \bar{r}_{-}=0$ at $t=0$ and so that $r$ satisfies (1.10), (1.11) for $t<0$.

By shifting the origin of time in the solution $z$ of Corollary 1, we obtain Corollary 2.

COROLlaRY 2. There is initial data $z(\gamma, 0)$, which is analytic in a neighborhood of $\gamma$ real, such that the solution $z(\gamma, t)$ of the Birkhoff-Rott equation (1.1) develops an infinite $(1+\nu)$ th derivative at a finite time $t_{0}$.

Finally we use the rescaling of $z$ to $z_{n}$. Take $z$ to be a solution for $t<0$ that develops an infinite $(1+\nu)$ th derivative at $t=0$, as in Corollary 1. Let $z_{N}(\gamma, t)=$ $N^{-2} z\left(N^{2} \gamma, N^{2} t-2 N\right)$ so that $s_{N}=z_{N}-\gamma=N^{-2} s\left(N^{2} \gamma, N^{2} t-2 N\right)$. Then at $t=0$ the $k$ th Sobolev norm of $s_{N}$ is bounded as

$$
\begin{aligned}
\left|s_{N}(\cdot, t=0)\right|_{H^{k}} & =N^{-2+2 k+3}|s(\cdot,-2 N)|_{H^{k}} \\
& \leqq N^{2 k+1} e^{-N} \\
& \rightarrow 0 \quad \text { as } N \rightarrow \infty .
\end{aligned}
$$

However, the time $T_{N}$ of singularity formation is $T_{N}=2 N^{-1} \rightarrow 0$ as $N \rightarrow \infty$. For $\nu>0$, denote $\sup \left|\partial_{\gamma}^{1+\nu} z\right|=\sup _{\gamma \neq \gamma^{\prime} \text { real }}\left|z^{\prime}(\gamma)-z^{\prime}\left(\gamma^{\prime}\right)\right| /\left|\gamma-\gamma^{\prime}\right|^{\nu}$. This shows the following.

COROllary 3. For any positive numbers $\nu, k, \varepsilon$, and $\delta$ there is initial data $z=\gamma+s_{0}$ with $|s|_{H^{k}}<\varepsilon$ such that $\sup \left|\partial_{\gamma}^{1+\nu} z\right|$ goes to infinity for $t=t_{0}<\delta$. In particular the initial value problem for the Birkhoff-Rott equation (1.1) is ill posed for any Sobolev space $H^{k}$ for $k>3 / 2$.

In other words, smallness of the initial perturbation $s$ is not sufficient to insure existence with bounded $(1+\nu)$ th derivative on any time interval for (1.1).

\section{REFERENCES}

[1] G. Birknoff, Helmholtz and Taylor instability in Hydrodynamic Instability, Proc. Sympos. in Appl. Math. XII, American Mathematical Society, Providence, RI, 1962, pp. 55-76.

[2] R. CAFlisCH AND O. OREllana, Long time existence for a slightly perturbed vortex sheet, Comm. Pure Appl. Math., 39 (1986), pp. 807-838.

[3] R. DiPERNA AND A. MAJDA, Oscillations and concentrations in weak solutions of the incompressible fluid equations, Comm. Math. Phys., 108 (1987), pp. 667-689.

[4] - Concentrations and regularization for 2-D incompressible flow, Comm. Pure Appl. Math., 40 (1987), pp. 301-345.

[5] — Reduced Hausdorff dimension and concentration cancellation for two-dimensional incompressible flow, J. Amer. Math. Soc., 1 (1988), pp. 59-86.

[6] J. DUCHON AND R. ROBERT, Solution globales avec nappe tourbillionaire pour les equations d'Euler dans le plan, C.R. Acad. Sci. Paris, 302 (1986), pp. 183-186.

[7] Y. Katznelson, An Introduction to Harmonic Analysis, Dover Press, New York, 1968.

[8] R. KRASNY, On singularity formation in a vortex sheet and the point vortex approximation, J. Fluid Mech., 167 (1986), pp. 65-93.

[9] - Desingularization of periodic vortex sheet roll-up, J. Comp. Phys., 65 (1986), pp. 292-313.

[10] D. I. Meiron, G. R. BAKer, ANd S. A. Orszag, Analytic structure of vortex sheet dynamics, Part 1, Kelvin-Helmholtz instability, J. Fluid Mech., 114 (1982), pp. 283-298.

[11] D. W. MOORE, The spontaneous appearance of a singularity in the shape of an evolving vortex sheet, Proc. Roy. Soc. London Ser. A, 365 (1979), pp. 105-119.

[12] - Numerical and analytical aspects of Helmholtz instability, in Theoretical and Applied Mechanics, Proc. XVI Internat. Congr. Theoret. Appl. Mech., F. I. Niordson and N. Olhoff, eds., North-Holland, Amsterdam, 1984, pp. 629-633.

[13] T. NishidA, A note on a theorem of Nirenberg, J. Differential Geometry, 12 (1977), pp. 629-633. 
[14] C. Sulem, P. L. Sulem, C. Bardos, AND U. Frisch, Finite time analyticity for the two and three dimensional Kelvin-Helmholtz instability, Comm. Math. Phys., 80 (1981), pp. 485-516.

[15] D. EBIN, Ill-posedness of the Rayleigh-Taylor and Helmholtz problems for incompressible fluids 13 (1988), pp. 1265-1295.

[16] J. DUChON AND R. Robert, Global vortex solutions of Euler equations in the plane, Comm. Partial Differential Equations, to appear. 